\title{
Functional outcomes and survival after surgical stabilization for inoperable non-small-cell lung cancer with spinal metastasis of the thoracic and lumbar spines: a retrospective comparison between epidermal growth factor receptor-tyrosine kinase inhibitor and platinum-based chemotherapy groups
}

\author{
Hsi-Hsien Lin $^{1,2} \cdot$ Chao-Hua Chiu ${ }^{1,3} \cdot$ Po-Hsin Chou ${ }^{1,2} \cdot$ Hsiao-Li Ma ${ }^{1,2} \cdot$ Jung-Pan Wang ${ }^{1,2} \cdot$ Shih-Tien Wang $\mathbb{D}^{1,2} \cdot$ \\ Chien-Lin Liu ${ }^{2} \cdot$ Ming-Chau Chang ${ }^{2}$
}

Received: 7 May 2019 / Revised: 29 July 2019 / Accepted: 24 August 2019 / Published online: 9 September 2019

(c) The Author(s), under exclusive licence to International Spinal Cord Society 2019

\begin{abstract}
Study design Retrospective cohort study.

Objectives To compare the surgical results between targeted therapy and post-operative chemotherapy for patients with spinal metastasis of inoperable non-small-cell lung cancer (NSCLC).

Setting Single-center study at an academic orthopedic department in Taiwan.

Methods Sixty-five patients were treated surgically for spinal metastasis of inoperable NSCLC with long posterior instrumentation with or without posterior decompression according to the patient's neurologic status. Post-operative radiotherapy of the spinal lesion and targeted therapy or chemotherapy were done following surgery after the surgical wound healed. Post-operative clinical outcomes and survival were evaluated and compared between these two groups. The overall survival represented survival from the date of diagnosis to death.

Results Thirty-five patients were grouped as the targeted therapy group and 30 patients as the chemotherapy group. The overall median survival times were 12.0 and 10.0 months in the targeted therapy and chemotherapy groups, respectively. Sixty-two patients were able to walk with or without an aid postoperatively. There was no significant difference observed between these two groups in terms of pain relief, neurologic improvement, ambulatory improvement, and survival.

Conclusions Surgical stabilization with or without laminectomy improved functional outcomes in patients with inoperable non-small-cell lung cancer, and post-operative functional outcomes were similar between chemotherapy and targeted therapy groups. A longer survival was observed with targeted therapy for the patients whose NSCLC was diagnosed before spinal metastasis, however, the longer survival was not statistically significant.
\end{abstract}

Shih-Tien Wang

stwang@vghtpe.gov.tw

1 School of Medicine, National Yang Ming University, Taipei, Taiwan

2 Department of Orthopedics and Traumatology, Taipei Veterans General Hospital, Taipei, Taiwan

3 Department of Chest Medicine, Taipei Veterans General Hospital, Taipei, Taiwan

\section{Introduction}

Metastatic spinal lesions can cause significant back pain and neurological morbidity and negatively impact quality of life [1] and survival. The role of surgery in the management of metastatic spinal cord compression is expanding [2]. Surgical treatment has been recommended for spinal metastasis associated with pain in noncompressive spinal lesions, multi-focal spinal disease, spinal metastatic disease with poor systemic control, and metastatic disease with impending mechanical instability [3], and has been reported associated with improved neurologic deficits and quality of life [4]. Moreover, the survivorship of spinal metastasis may be influenced by a 
newly developed chemotherapy, which targets specific gene mutations [5].

Non-small-cell lung cancer (NSCLC) cases comprise $\sim 85 \%$ of lung cancer cases, and the spinal column has been reported as the most common site of extrapulmonary metastasis [6]. Patients with advanced and chemotherapyrefractory NSCLC have been treated with gefitinib or erlotinib, both epidermal growth factor receptor-tyrosine kinase inhibitors (EGFR-TKI). Additionally, EGFR-TKI has been used with proven clinical efficacy for medical treatment of NSCLC since 2004 [7]. There were, however, few reports on the results of surgery for the spinal metastasis of inoperable NSCLS. Furthermore, comparisons between the efficacy of the new targeted therapies and traditional platinum-based chemotherapy as the post-operative adjuvant therapy for spinal metastasis were sparse. Therefore, we conducted a retrospective study to evaluate and compare the surgical results of inoperable NSCLC patients with spinal metastasis between targeted therapy and traditional platinum-based chemotherapy in terms of pain relief, improvement of neurologic deficits, improvement of ambulatory ability and survival. Survival for the patients with inoperable NSCLC who were diagnosed before experiencing spinal metastasis between targeted therapy and platinum-based chemotherapy was also evaluated.

\section{Methods}

From May 2001 to August 2011, 166 patients in our institute with spinal metastasis of thoracic and lumbar spine underwent posterior long instrumentation with or without posterior decompression depending on neurologic status. Among them, 65 patients with primary inoperable NSCLC were included in this study. Their medical records were reviewed, and the patients were followed up until their death. The research protocol was approved by the institutional review board at our hospital. Routine evaluation including chest computerized tomography (CT), brain magnetic resonance imaging (MRI), and whole- body bone scan. Whole body positron emission tomography (PET) scan was not covered by National Health Insurance. Chest CT was used to evaluate visceral metastasis by scanning from neck to upper kidney. If there were other suspicious symptoms, CT or MRI over the region was performed. The indications for surgery were an expected survival time of $>3$ months, impending mechanical instability with more than a 50\% involvement of the vertebrae, pathologic fracture of the involved vertebrae, neurological deficit, and for establishment of a tissue diagnosis. Patients with evident neurologic signs caused by brain metastasis, multiple visceral metastasis, an expected survival time $<3$ months, multilevel spinal cord compression due to tumor, poor general condition, and poor compliance, who were not suitable for surgical treatment, were excluded. Multilevel spinal metastasis without spinal cord compression and brain metastasis without a neurologic deficit and with an expected survival time $>3$ months were not contraindications.

Radiologic evaluations before operation included plain anteroposterior and lateral radiographs, magnetic resonance imaging (MRI), whole-body bone scan, and computerized tomography (CT)-guided biopsy for those patients without neurologic deficit. For those patients with neurologic deficits who could not wait for CT-guided biopsy, intraoperative biopsy was done during surgery. To reduce intraoperative blood loss, angiography with embolization of the tumor vessels was routinely performed before surgery.

Long posterior instrumentation two levels above and two levels below the lesion was carried out with or without posterior decompression depending on whether the patient had neurologic deficits. All patients were operated on by the same spinal surgeons in our hospital with similar transpedicle screw and hook systems. Hooks were used on the metastasized vertebrae instead of transpedicle screws because good bony purchase could not be achieved by transpedicle screws in osteolytic vertebrae. Post operation, after removal of drainage, patients were allowed to ambulate in a brace, which was used for 3-6 months after the operation until there was bony regeneration of the involved vertebrae without back pain. Post-operative radiotherapy on the lesion level was arranged 2 weeks after surgery or after wound healing by a radiation oncologist and followed by platinum-based chemotherapy or targeted therapy with EGFR-TKI, decided by the physicians of the Department of Chest Medicine at our hospital.

Every patient was followed with serial anteroposterior and lateral radiographs, immediately after surgery and at post-operative 6 weeks, 3, 6, and 12 months, and then annually. Bony regeneration of the metastasized vertebra was defined as regeneration of the destructed bone without evidence of recurrent osteolysis on plain film.

According to the policy of Taiwan's National Health Insurance before 2011, all of the patients with metastatic NSCLC should receive platinum-based chemotherapy as first-line treatment $[8,9]$, and could be crossed over to EGFR-TKI if they experienced disease progression. As there was no solid evidence about which drug was better in efficacy, clinical decisions regarding patients' physical status, resources, insurances, and values were made by the physicians of the Department of Chest Medicine at our hospital. In this study, 35 patients who received EGFR-TKI till death were grouped in the targeted therapy group (Fig. 1), 20 patients received gefitinib, and 15 used erlotinib. Only six patients received gene mutation detection of epidermal growth factor receptor, which was not available until 2009 at our hospital, before the use of targeted therapy. Another 30 patients were included in the chemotherapy group (Fig. 2). 


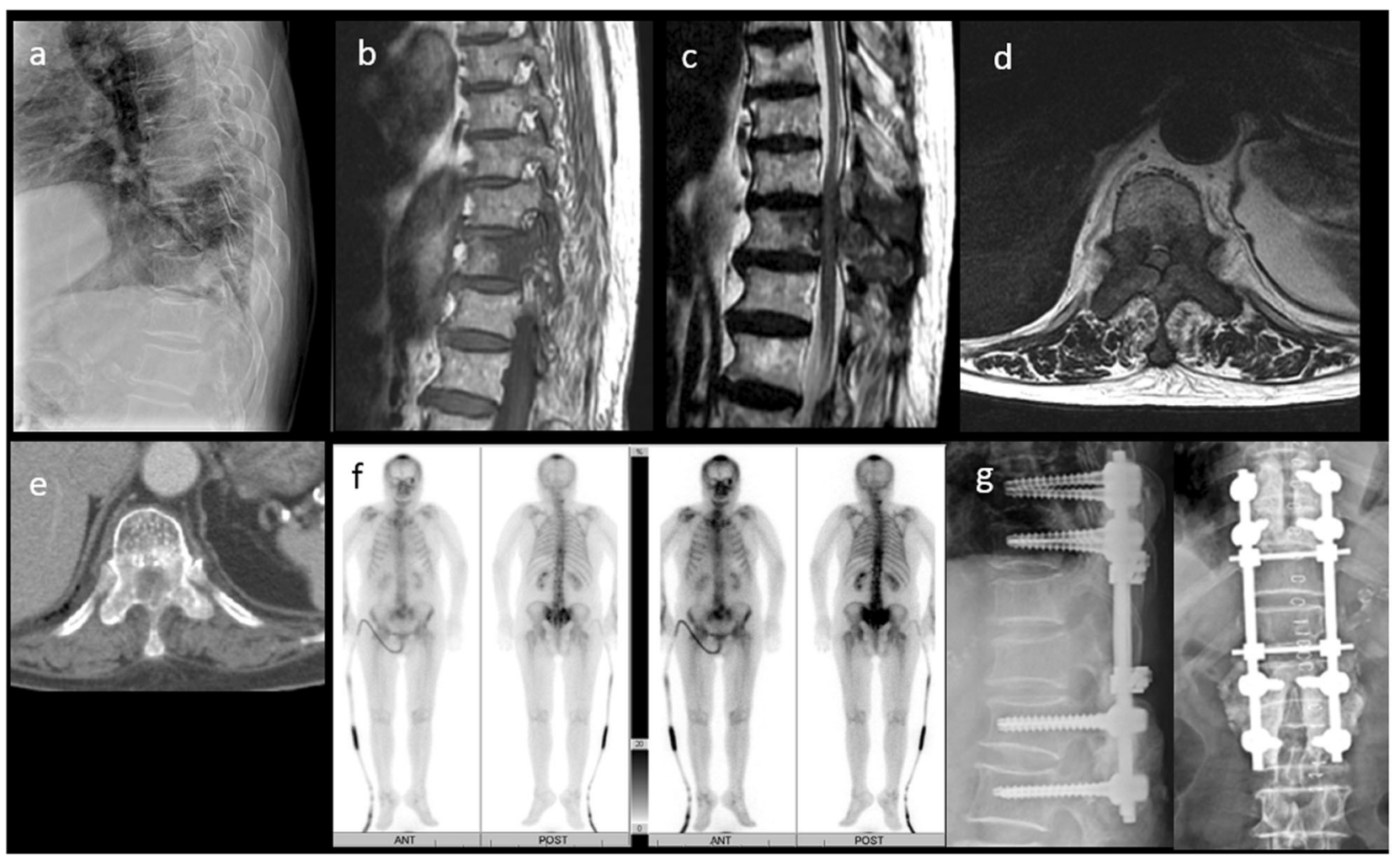

Fig. 1 Eighty-four-year-old female in the target therapy group. a Preoperative plain film. b Preoperative sagittal T1-weighted MRI. c Preoperative sagittal T2-weighted MRI. d Preoperative axial t2- weighted MRI. e Preoperative CT scan. f Whole-body bone scan. g Post-operative anteroposterior and lateral plain film

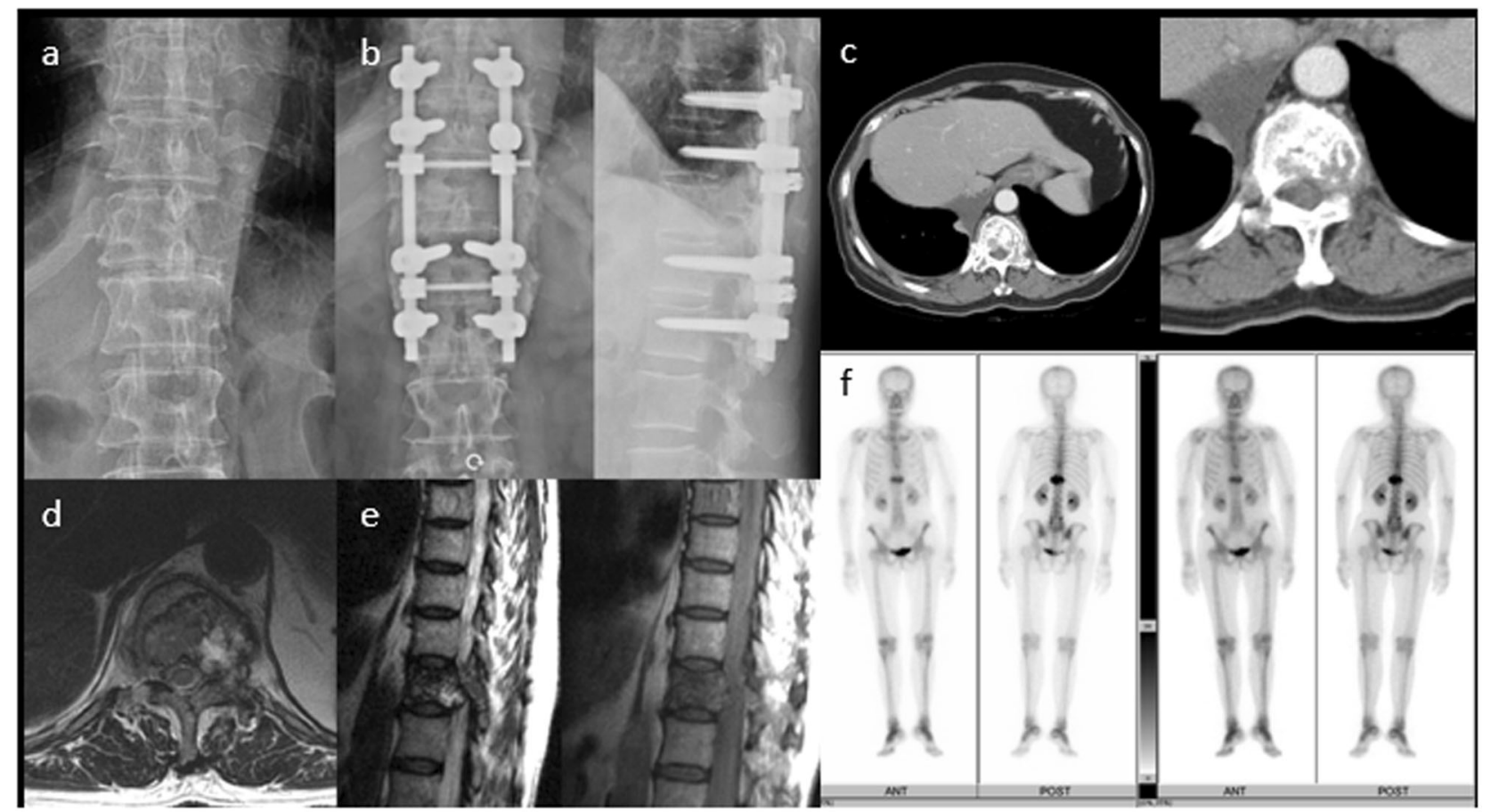

Fig. 2 Sixty-five-year-old female in the chemotherapy group. a Preoperative plain film. b Post-operative anteroposterior and lateral plain film. c Preoperative CT scan. d Preoperative axial MRI. e Preoperative sagittal T1- and T2-weighted MRI. f Whole-body bone scan. The $x$-axis is the timeline and the $y$-axis is the survival probability 
Table 1 NSCLC patients with spinal metastasis

\begin{tabular}{llll}
\hline & Overall & Targeted therapy group & Chemotherapy group \\
\hline Age & & & \\
$\quad$ Mean \pm SD (range) & $\begin{array}{l}60.7 \pm 11.3 \\
(36-84)\end{array}$ & $55.1 \pm 9.5(42-84)$ & $67.0 \pm 9.5(36-80)$ \\
Male:female & $39: 26$ & $19: 16$ & $20: 10$ \\
Diagnosis of NSCLC & 65 & 35 & 30 \\
Before spinal metastasis & 39 & 21 & 18 \\
After spinal metastasis & 26 & 14 & 12 \\
Surgical removal of the & 0 & 0 & 0 \\
primary NSCLC & & & $1.3 \pm 0.7(1-4)$ \\
Number of involved segments & $1.6 \pm 1.0(1-5)$ & $1.8 \pm 1.1(1-5)$ & 24 \\
$\quad$ Mean \pm SD (range) & 44 & 20 & 6 \\
$\quad$ Single level & 21 & 15 & \\
$\quad$ Multiple level & rand & &
\end{tabular}

$N S C L C$ non-small-cell lung cancer, $S D$ standard deviation
Post-operative clinical outcomes were evaluated with neurologic status, ambulatory ability, visual analog scale (VAS) for pain, and survival time. Neurological deficits were graded according to the Frankel classification system before and after surgery. Ambulatory abilities were graded as bedridden, wheel chair-bound, with an aid (walker, cane, crutches, or needing assistance), and without an aid, and were recorded for every patient before and after operation. Post-operative survival time was calculated from the day of surgery.

Survival curves were calculated using the Kaplan-Meier method with Stata Statistical Software, release 12 (StataCorp LP, College Station, TX), and the significance of the difference between groups was compared. The overall survival represented the time from the date of diagnosis to death. Statistical analysis of the survival time and VAS for pain was performed using the unpaired $t$-test and Mann-Whitney Rank Sum test from the SigmaStat software package, version 2.0 (Jandel Corp., San Rafael, CA). A $P$-value $<0.05$ was considered significant. To determine whether these tests were sufficiently powered, power analysis was also performed with $\alpha$ set at 0.05 , using the SigmaStat software package, version 2.0.

Cox regression analysis was used to investigate whether demographic variables regarding age, gender, NSCLC diagnosis timing and number of spinal involvement might be confounders for overall survivorship.

\section{Results}

All 65 patients, 39 males and 26 females, were completely followed up until their death. The mean age at diagnosis of NSCLC was $60.7 \pm 11.3$ years old (range, 36-84-years-old). None of these patients received surgical removal of the primary NSCLC. There was no significant difference observed in patients' age, gender, and the number of metastasized vertebral bodies between the targeted therapy group and the chemotherapy group (Table 1).

The median overall survival times for all of the patients were 11.0 months (range, 1-55 months), 12.0 months (range, 1-45 months) for the targeted therapy group, and 10.0 months (range, 1-55 months) for the chemotherapy group. The median post-operative survival times for all of the patients were 7.0 months (range, 1-35 months), 6.0 months (range, 1-24 months) for the targeted therapy group, and 7.5 months (range, 1-35 months) for the chemotherapy group (Table 2). There was no statistical significance observed on survival time between these two groups for overall (Fig. 3) and post-operative (Fig. 4) survival times. Moreover, the survival effects in these two groups were not correlated with the demographic variables regarding age, gender, NSCLC diagnosis timing, and number of spinal involvement, based on our Cox regression analysis.

Thirty-nine patients were diagnosed to have inoperable NSCLC before spinal metastasis; 21 patients were in the targeted therapy group and 18 were in the chemotherapy group, with median 6.0-month (range, 1-24 months) and 6.5-month (ranged, 2-25 months) post-operative survival time, respectively (Table 2 and Fig. 5). The median overall survival times for these patients were 14.0 months (range, 4-45 months) and 11.5 months (range, 6-55 months), respectively, for the targeted therapy and the chemotherapy groups (Table 2 and Fig. 6). Although there was a trend of longer overall median survival time in patients who received EGFR-TKI before being symptomatic with spinal metastasis, there was no statistical significant difference observed comparing with patients receiving platinum-based chemotherapy (Fig. 6).

The remaining 26 patients were diagnosed to have inoperable NSCLC after spinal metastasis; 14 patients were 
Table 2 Clinical outcomes for the inoperable NSCLC patients with spinal metastasis after operation

\begin{tabular}{lllll}
\hline & Overall & Targeted therapy group & Chemotherapy group & $P$ \\
\hline VAS [mean \pm SD (range)] & & & & \\
$\quad$ Preoperative VAS & $6.8 \pm$ & $6.5 \pm 1.0(3-8)$ & $7.0 \pm 1.0(3-8)$ & 0.15 \\
& $0.5(3-8)$ & & & \\
Post-operative VAS & $3.8 \pm$ & $3.7 \pm 1.2(1-6)$ & $3.8 \pm 1.3(2-6)$ & 0.12 \\
& $1.3(1-6)$ & & & \\
Survival time [Median months (range)] & & & \\
Overall & $11.0(1-55)$ & $12.0(1-45)$ & $11.5(6-55)$ & $0.0(1-55)$ \\
NSCLC before spinal metastasis & $13.0(1-55)$ & $14.0(4-45)$ & $7.5(1-35)$ & 0.61 \\
Post-operative survival & $7.0(1-35)$ & $6.0(1-24)$ & $6.5(2-25)$ & 0.40 \\
NSCLC before spinal metastasis & $7.0(1-25)$ & $6.0(1-24)$ & $7.0(1-35)$ & 0.56 \\
NSCLC after spinal metastasis & $6.0(1-35)$ & $5.5(1-24)$ & & \\
\hline
\end{tabular}

NSCLC non-small-cell lung cancer, VAS visual analog scale for pain, $S D$ standard deviation

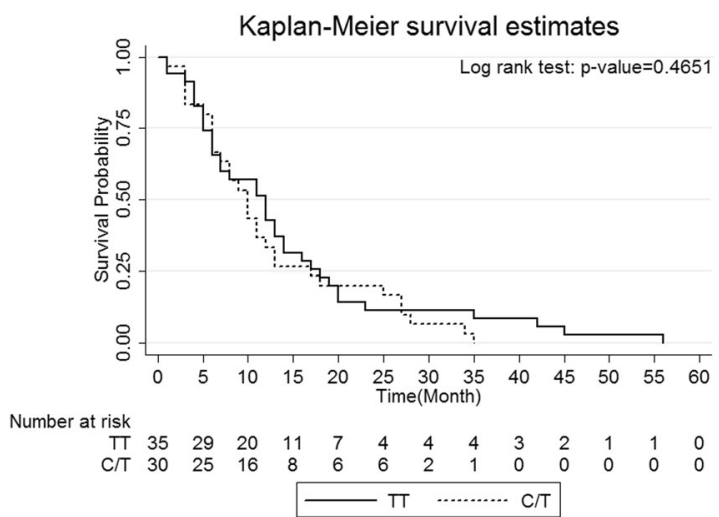

Fig. 3 Kaplan-Meier analysis revealed no significance difference in overall survival for the patients with chemotherapy or targeted therapy

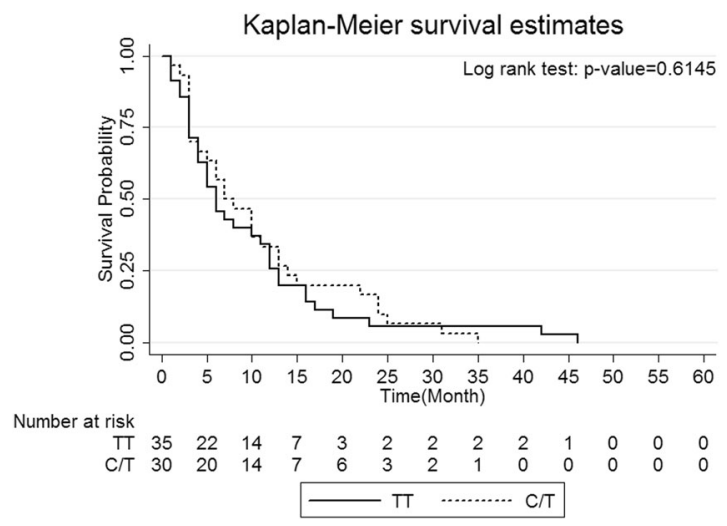

Fig. 4 Kaplan-Meier analysis revealed no significance difference in post-operative survival for the patients with chemotherapy or targeted therapy

in the targeted therapy group, with a median 5.5-month survival time (range, 1-24 months), 12 patients were in the chemotherapy group, with a median 7.0-month survival time (ranged, 1-35 months). There was no statistically significant difference observed in the survival time between

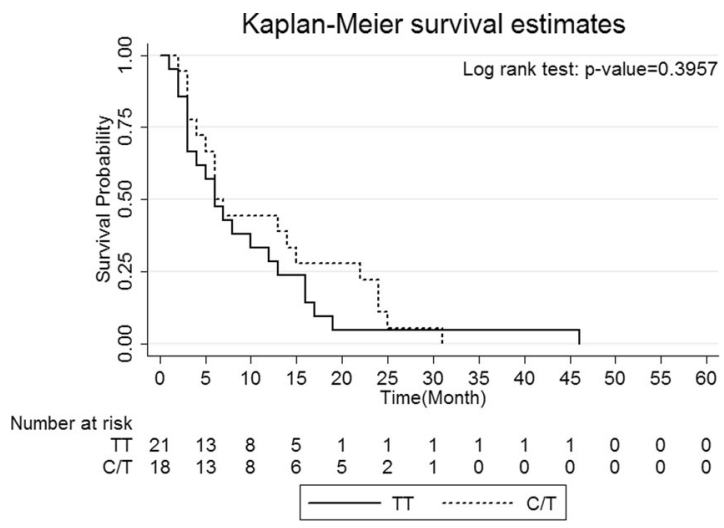

Fig. 5 Kaplan-Meier analysis revealed no significance difference in post-operative survival for the patients receiving chemotherapy or targeted therapy before the diagnosis of spinal metastasis

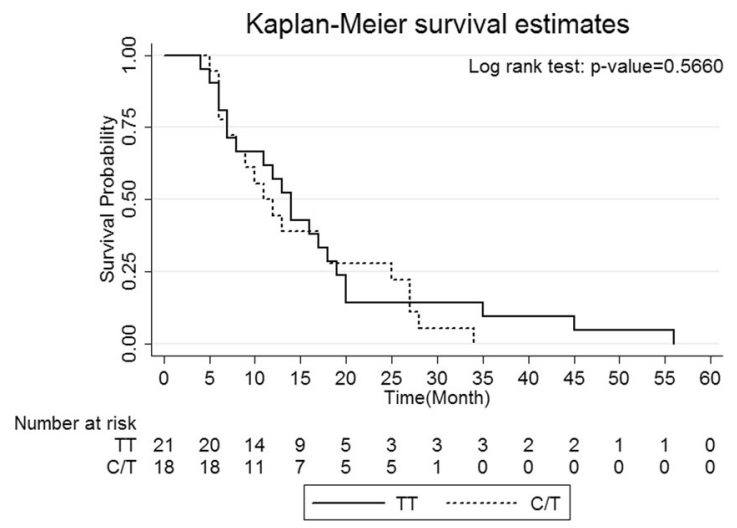

Fig. 6 Kaplan-Meier analysis revealed no significance difference in overall survival for the patients receiving chemotherapy or targeted therapy before the diagnosis of spinal metastasis

these two groups, no matter whether the diagnosis of inoperable NSCLS was made before or after spinal metastasis (Table 2).

The average VAS for pain was $6.8 \pm 0.5$ (range, 3-8) preoperatively, and was significantly improved to an 
average of $3.8 \pm 1.3$ (range, 1-6) at patient's discharge or 2 weeks after surgery (Table 2). According to the Frankel grading system, 33 patients had various degrees of neurologic deficits preoperatively, with an incidence of $50.8 \%$; one patient was Frankel A, two patients were Frankel B, 13 patients were Frankel C, and 17 patients were Frankel D (Table 3a, b, c). After operation, however, ten patients remained unchanged as Frankel $\mathrm{C}$ and $\mathrm{D}$ preoperatively, and $1(3.0 \%)$ patient deteriorated from Frankel D to Frankel $\mathrm{C}$ due to brain metastasis, which was found after operation.

Preoperatively, 62 patients had various degrees of ambulatory deficit, with an incidence of $95.4 \%$; three patients were bedridden, 19 patients were wheel chairbound, and 40 patients were ambulatory with aid by various

Table 3a Neurological status before and after operation by Frankel's classification

\begin{tabular}{lllllll}
\hline Preoperative & \multicolumn{3}{l}{ Post-operative } & \multirow{2}{*}{ Total } \\
\cline { 2 - 5 } & $A$ & $B$ & $C$ & $D$ & $E$ & \\
\hline$A$ & 0 & 0 & 1 & 0 & 0 & 1 \\
$B$ & 0 & 0 & 2 & 0 & 0 & 2 \\
$C$ & 0 & 0 & 2 & 10 & 1 & 13 \\
$D$ & 0 & 0 & 1 & 8 & 8 & 17 \\
$E$ & 0 & 0 & 0 & 0 & 32 & 32 \\
Total & 0 & 0 & 6 & 18 & 41 & 65 \\
\hline
\end{tabular}

Table 3b Neurological status before and after operation by Frankel's classification in the chemotherapy group

\begin{tabular}{lllllll}
\hline Preoperative & \multicolumn{3}{l}{ Post-operative } & \multirow{2}{*}{ Total } \\
\cline { 2 - 5 } & $A$ & $B$ & $C$ & $D$ & $E$ & \\
\hline$A$ & 0 & 0 & 1 & 0 & 0 & 1 \\
$B$ & 0 & 0 & 1 & 0 & 0 & 1 \\
$C$ & 0 & 0 & 1 & 6 & 1 & 8 \\
$D$ & 0 & 0 & 1 & 2 & 2 & 5 \\
$E$ & 0 & 0 & 0 & 0 & 15 & 15 \\
Total & 0 & 0 & 4 & 8 & 18 & 30 \\
\hline
\end{tabular}

Table 3c Neurological status before and after operation by Frankel's classification in the targeted therapy group

\begin{tabular}{lllllll}
\hline Preoperative & \multicolumn{3}{l}{ Post-operative } & Total \\
\cline { 2 - 5 } & $A$ & $B$ & $C$ & $D$ & $E$ & \\
\hline$A$ & 0 & 0 & 0 & 0 & 0 & 0 \\
$B$ & 0 & 0 & 1 & 0 & 0 & 1 \\
$C$ & 0 & 0 & 1 & 4 & 0 & 5 \\
$D$ & 0 & 0 & 0 & 6 & 6 & 12 \\
$E$ & 0 & 0 & 0 & 0 & 17 & 17 \\
Total & 0 & 0 & 2 & 10 & 23 & 35 \\
\hline
\end{tabular}

methods (Table 4a). After operation, 62 patients had an average 1.2 grade improvement, average 1.1 grade for the chemotherapy group (Table $4 \mathrm{~b}$ ) and 1.3 grade for the targeted therapy group (Table 4c). None of the patients was bedridden after surgery. The post-operative clinical outcomes and overall survival were similar between these two groups, but the statistical power was not enough to draw the conclusion.

Bony regeneration was found in all of the patients without any sign of local recurrence until their death. There was no intra-operative mortality. Six patients had surgeryrelated complications, including one with wound dehiscence after radiotherapy and five with superficial wound infection. All were successfully managed by conservative treatment. One patient had pyogenic vertebral osteomyelitis by methicillin-resistant Staphylococcus aureus proved by CT-guided biopsy and was successfully managed conservatively with intravenous antibiotics for 6 weeks followed by a 6-week course of oral antibiotics.

\section{Discussion}

With recent advances in targeted therapies, the life expectancy of NSCLC patients can be prolonged even if there is poor performance status [2]. It is believed that EGFR-TKI inhibits tumor cell proliferation and induces normal bone formation [10]. Although bony metastases clearly reflect the existence of widespread disease, it is necessary to specifically evaluate the efficacy of new therapies for spinal metastasis of inoperable NSCLC. However, reports concerning the surgical results of spinal metastases of lung cancer patients are few in number.

Prognostic factors of survival after surgical treatment of spinal metastasis of NSCLC are thought to be related with gender, preoperative performance status, calcium, albumin [11], pathologic fracture [12], and post-operative ambulatory status [13]. The most emphasized significant factors are visceral metastases [14] and post-operative ambulatory status [15], despite it being believed that control of systemic disease does not predict duration of post-operative survival [16]. In our study, evident neurologic deficits caused by brain metastases were excluded due to patients' poor general condition and poor compliance. However, multilevel spinal metastasis without spinal cord compression and brain metastasis without a neurologic deficit and with an expected survival time of $>3$ months were not contraindications. In our study, the overall median post-operative survival time for spinal metastasis of inoperable NSCLC was 7 months, 6 months with targeted therapy and 7.5 months with platinum-based chemotherapy, and there was no statistical significance observed between these two regimens of treatment (Table 2 and Fig. 4). 
Table 4a Ambulatory ability before and after operation

\begin{tabular}{llllll}
\hline & \multicolumn{2}{l}{ Post-operative } & Total \\
\cline { 2 - 5 } & Bedridden & Wheel chair-bound & With an aid & Without an aid & \\
\hline Preoperative & 0 & 2 & 1 & 0 & 3 \\
Bedridden & 0 & 1 & 4 & 14 & 19 \\
Wheel chair-bound & 0 & 0 & 2 & 38 & 40 \\
With an aid & 0 & 0 & 0 & 3 & 3 \\
Without an aid & 0 & 3 & 7 & 55 & 65 \\
Total & 0 & & & & \\
\hline
\end{tabular}

\begin{tabular}{llllll}
\hline & \multicolumn{2}{l}{ Post-operative } & Total \\
\cline { 2 - 5 } & Bedridden & Wheel chair-bound & With an aid & Without an aid & \\
\hline Preoperative & 0 & 1 & 1 & 0 & 2 \\
Bedridden & 0 & 1 & 4 & 5 & 10 \\
Wheel chair-bound & 0 & 0 & 1 & 16 & 17 \\
With an aid & 0 & 0 & 0 & 1 & 1 \\
Without an aid & 0 & 2 & 6 & 22 & 30 \\
Total & 0 & & & & \\
\hline
\end{tabular}

\begin{tabular}{llllll}
\hline & \multicolumn{2}{l}{ Post-operative } & & Total \\
\cline { 2 - 5 } & Bedridden & Wheel chair-bound & With an aid & Without an aid & \\
\hline Preoperative & 0 & 1 & 0 & 0 & 1 \\
Bedridden & 0 & 0 & 0 & 9 & 9 \\
Wheel chair-bound & 0 & 0 & 1 & 22 & 23 \\
With an aid & 0 & 0 & 0 & 2 & 35 \\
Without an aid & 0 & 1 & 1 & 33 & 35 \\
Total & 0 & & & & \\
\hline
\end{tabular}

Table 4c Ambulatory ability before and after operation in the targeted therapy group
Table 4b Ambulatory ability before and after operation in the chemotherapy group

\section{(a)}

Patients with NSCLC spinal metastases have an estimated median post-operative survival time of 8.8 months regardless of the type of chemotherapy [17], and overall survival was improved to 10.5 months with targeted therapy [18], which is similar to the results of the current study, which were 12.0 months for the targeted therapy group and 10.0 months for the chemotherapy group (Table 2 and Fig. 3). For the patients who were diagnosed as having inoperable NSCLC before spinal metastasis, the overall median survival times were 14 months with targeted therapy and 11.5 months for chemotherapy, with no statistical significance observed between those two groups (Table 2 and Fig. 6). For patients who were diagnosed with inoperable NSCLC after spinal metastasis, the median survival times for the targeted therapy group were 5.5 months and 7.0 months for the chemotherapy group; no statistical significance was observed between the two groups (Table 2).
EGFR mutations are not related to skeleton-related event patterns in patients with lung adenocarcinoma [19]. More aggressive treatment may be chosen when there are two prognostic characteristics: adenocarcinoma and an EGFR mutation [20]. Potent and continuous relief of spinal bone pain due to metastasis of NSCLC by treatment with EGFR-TKI alone has been reported [21]. However, in cases in which the primary tumors show EGFR mutation, the corresponding metastatic tumors may not show EGFR mutation; a 27\% discordance rate was found in 67 paired tissue samples (primary and metastatic tumors) using the Scorpion Amplified Refractory Mutation System assay [6]. Therefore, EGFR-TKI treatment may not have the same effects on primary and metastatic tumors. This may explain the reason why the survival time of our study was not statistically different between targeted therapy and chemotherapy after surgical treatment of spinal 
metastasis. Overall survival was noted to be improved with EGFR-TKI treatment for patients with EGFR mutations [22]. However, gene detection was not available in the initial period of the study, which might have made the results similar between those two groups. Additionally, a high crossover rate of $53.8 \%$ from chemotherapy to EGFR-TKI therapy was noted in our study, especially when there was disease progression following traditional platinum-based chemotherapy, which is the first-line chemotherapy according to national health insurance policy in our country. This is inevitable, since the optimal treatment of NSCLC is unclear and there is no defined standard therapy [23]. Further subgroups should be analyzed separately in future trials.

The benefits of palliative surgery for bony metastasis of the spine include reduction of pain, improvement of neurologic deficit, avoiding the morbidity of prolonged bedridden status, and improved nursing care [24]. However, it is difficult to identify those patients who will benefit from palliative surgery [25]. Moreover, evaluating cancer patients as candidates for major spine surgery must be done in the context of prognosis [26]. Although bony metastases and their subsequent surgical treatment in lung cancer patients are associated with high morbidity and mortality [27], surgical treatment is necessary under certain circumstances, including impending mechanical instability with $>50 \%$ destruction of the vertebra and one pedicle, pathologic fracture of the involved vertebra, neurological deficit due to spinal cord compression [28], and for establishment of a tissue diagnosis for those patients with an expected survival time longer than 3 months [29]. In our study, the VAS for pain was improved from 6.8 to 3.8 , and the improvement of neurologic deficit was at least one Frankel grade for the 22 out of the $33(67 \%)$ patients with neurologic deficit. However, ten patients $(30.3 \%)$ remained no change, and one $(3.0 \%)$ patient deteriorated from Frankel D to Frankel $\mathrm{C}$ due to brain metastasis, which was found after operation. Ambulatory ability was improved for 62 (95.4\%) patients with various ambulatory deficits, and none was bedridden. No statistical difference was observed between these two therapy groups in terms of VAS for pain, neurologic and ambulatory improvement.

Adjuvant therapy with radiotherapy or chemotherapy after surgical treatment is effective for the local control of spinal lesion [30]. In our study, post-operative radiotherapy was routinely performed, and no local recurrence was found for all of the patients until their death.

This study had several weaknesses or drawbacks. First of all, there were not enough patients enrolled to make a conclusion with sufficient statistical power. That this study failed to find differences may merely be due to an insufficient sample size. Also, we had to consider pooling data with other institutions to address the surgical outcomes and post-operative effects of targeted therapy on the overall survivorship in the future. Second, we did not check the concordance of EGFR mutations between primary NSCLC and metastatic tumors, which may play a very important role in explaining the similar survival after surgical stabilization between these two groups. Third, we used Frankel classification instead of International Standards for Neurological Classification of Spinal Cord Injury (ISNCSI) to evaluate the neurological deficits because Frankel classification is more simple and easy to use and has been used in many studies. Fourth, the retrospective and single-center nature of this study was also a major drawback. Therefore, a larger-scale, multi-center prospective study is needed with same level and the extent of spinal metastases, detailed chemotherapy regimens, and tumor cell gene mutation test in the future to determine whether EGFR-TKI is effective for inoperable NSCLC patients with spinal metastasis after surgical intervention.

In conclusion, surgical stabilization for patients with spinal metastasis of inoperable NSCLC improved clinical outcome in terms of VAS for pain, ambulatory ability, and neurologic improvement, although there was no difference between targeted therapy and chemotherapy. For patients receiving targeted therapy with EGFR-TKI, the postoperative survivorship was not significantly longer than that for those patients treated with platinum-based chemotherapy, irrespective of diagnosis of spinal metastasis before or after diagnosis of NSCLC. Although a longer overall survivorship in patients receiving EGFR-TKI was observed for patients whose NSCLC was diagnosed before spinal metastasis, the longer survivorship was not statistically significant. Discordance of gene expression between primary and metastatic tissue may play a role to explain the phenomenon with our initial exploratory data, however, further study in the future is needed.

\section{Data archiving}

The data sets generated and analyzed during the current study are available from the corresponding author on reasonable request.

Acknowledgements The study was supported by Division of Spine Surgery, Department of Orthopedics and Traumatology, Taipei Veterans General Hospital. We thank Hsin-Yi Huang from the Biostatistics Task Force, Taipei Veterans General Hospital, for the statistical assistance.

Author contributions H-HL: Data curation; formal analysis; investigation; software; writing - original draft. C-HC: conceptualization; data curation; investigation. P-HC, H-LM, J-PW: data curation; investigation; software. S-TW: conceptualization, validation; writing -review \& editing. C-LL, M-CC: supervision. 


\section{Compliance with ethical standards}

Conflict of interest The authors declare that they have no conflict of interest.

Ethical approval The study protocol was approved and supervised by the Institutional Review Board of Taipei Veterans General Hospital.

Publisher's note Springer Nature remains neutral with regard to jurisdictional claims in published maps and institutional affiliations.

\section{References}

1. Maccauro G, Spinelli MS, Mauro S, Perisano C, Graci C, Rosa MA. Physiopathology of spine metastasis. Int J Surg Oncol. 2011;2011:107969.

2. Schmidt MH, Klimo P Jr., Vrionis FD. Metastatic spinal cord compression. J Natl Compr Canc Netw. 2005;3:711-9.

3. Oliveira MF, Barros Bde A, Rotta JM, Botelho RV. Tokuhashi Scoring System has limited applicability in the majority of patients with spinal cord compression secondary to vertebral metastasis. Arq Neuropsiquiatr. 2013;71:798-801.

4. Patchell RA, Tibbs PA, Regine WF, Payne R, Saris S, Kryscio RJ, et al. Direct decompressive surgical resection in the treatment of spinal cord compression caused by metastatic cancer: a randomised trial. Lancet. 2005;366:643-8.

5. Yan W, Xiao J, Liu T, Huang W, Yang X, Wu Z, et al. The effects of Hsp90 expression alteration on spinal metastases of breast carcinoma. Tumour Biol. 2013;34:1391-7.

6. Chang SC, Chang CY, Shih JY. The role of epidermal growth factor receptor mutations and epidermal growth factor receptortyrosine kinase inhibitors in the treatment of lung cancer. Cancers (Basel). 2011;3:2667-78.

7. Paez JG, Janne PA, Lee JC, Tracy S, Greulich H, Gabriel S, et al. EGFR mutations in lung cancer: correlation with clinical response to gefitinib therapy. Science. 2004;304:1497-1500.

8. Park JO, Kim SW, Ahn JS, Suh C, Lee JS, Jang JS, et al. Phase III trial of two versus four additional cycles in patients who are nonprogressive after two cycles of platinum-based chemotherapy in non small-cell lung cancer. J Clin Oncol. 2007;25:5233-9.

9. Ettinger DS, Akerley W, Borghaei H, Chang AC, Cheney RT, Chirieac LR, et al. Non-small cell lung cancer, version 2.2013. J Natl Compr Canc Netw. 2013;11:645-53.

10. Chang GC, Chen KC, Yang TY, Yin MC, Lin CP, Kuo BI, et al. Activity of gefitinib in advanced non-small-cell lung cancer with very poor performance status. Invest New Drugs. 2005;23:73-7.

11. Ogihara S, Seichi A, Hozumi T, Oka H, Ieki R, Nakamura K, et al. Prognostic factors for patients with spinal metastases from lung cancer. Spine (Philos Pa 1976). 2006;31:1585-90.

12. Wibmer C, Leithner A, Hofmann G, Clar H, Kapitan M, Berghold A, et al. Survival analysis of 254 patients after manifestation of spinal metastases: evaluation of seven preoperative scoring systems. Spine (Philos Pa 1976). 2011;36:1977-86.

13. Moon KY, Chung CK, Jahng TA, Kim HJ, Kim CH. Postoperative survival and ambulatory outcome in metastatic spinal tumors: prognostic factor analysis. J Korean Neurosurg Soc. 2011;50:216-23.
14. Leithner A, Radl R, Gruber G, Hochegger M, Leithner K, Welkerling $\mathrm{H}$, et al. Predictive value of seven preoperative prognostic scoring systems for spinal metastases. Eur Spine J. 2008;17:1488-95.

15. Yang SB, Cho W, Chang UK. Analysis of prognostic factors relating to postoperative survival in spinal metastases. J Korean Neurosurg Soc. 2012;51:127-34.

16. Walcott BP, Cvetanovich GL, Barnard ZR, Nahed BV, Kahle KT, Curry WT. Surgical treatment and outcomes of metastatic breast cancer to the spine. J Clin Neurosci. 2011;18:1336-69.

17. Chen YJ, Chang GC, Chen HT, Yang TY, Kuo BI, Hsu HC, et al. Surgical results of metastatic spinal cord compression secondary to non-small cell lung cancer. Spine (Philos Pa 1976). 2007;32: E413-8.

18. Yang ZY, Liu L, Mao C, Wu XY, Huang YF, Hu XF, et al. Chemotherapy with cetuximab versus chemotherapy alone for chemotherapy-naive advanced non-small cell lung cancer. Cochrane Database Syst Rev. 2014;11:CD009948.

19. Nagata M, Kudoh S, Mitsuoka S, Suzumura T, Umekawa K, Tanaka H, et al. Skeletal-related events in advanced lung adenocarcinoma patients evaluated EGFR mutations. Osaka City Med J. 2013;59:45-52.

20. Aoude AA, Amiot LP. Surgical treatment of a patient with lung cancer metastasized to the spine with EGFR mutation: A case report. Int J Surg Case Rep. 2012;3:510-2.

21. Kanda S, Fujimoto T, Ito T, Mori S. [Potent and continuous relief of spinal bone pain due to metastasis of non-small cell lung cancer by treatment with gefitinib-a case report]. Gan Kagaku Ryoho. 2009;36:803-5.

22. Yang JC, Wu YL, Schuler M, Sebastian M, Popat S, Yamamoto $\mathrm{N}$, et al. Afatinib versus cisplatin-based chemotherapy for EGFR mutation-positive lung adenocarcinoma (LUX-Lung 3 and LUXLung 6): analysis of overall survival data from two randomised, phase 3 trials. Lancet Oncol. 2015;16:141-51.

23. Stinchcombe TE. Novel agents in development for advanced nonsmall cell lung cancer. Ther Adv Med Oncol. 2014;6:240-53.

24. Putz C, Gantz S, Bruckner T, Moradi B, Helbig L, Gerner HJ, et al. Preoperative scoring and limits of prognostication: functional outcome after surgical decompression in metastatic spinal cord compression. Oncology. 2014;86:177-1784.

25. Weiss RJ, Wedin R. Surgery for skeletal metastases in lung cancer. Acta Orthop. 2011;82:96-101.

26. Tokuhashi Y, Matsuzaki H, Oda H, Oshima M, Ryu J. A revised scoring system for preoperative evaluation of metastatic spine tumor prognosis. Spine (Philos Pa 1976). 2005;30:2186-91.

27. Cansever T, Kabatas S, Civelek E, Yilmaz C, Caner H. Spinal metastasis of occult lung carcinoma causing cauda equine syndrome with lumbar spinal stenosis. Turk Neurosurg. 2011;21:408-12.

28. Putz C, Wiedenhofer B, Gerner HJ, Furstenberg CH. Tokuhashi prognosis score: an important tool in prediction of the neurological outcome in metastatic spinal cord compression: a retrospective clinical study. Spine (Philos Pa 1976). 2008;33:2669-74.

29. Choi D, Crockard A, Bunger C, Harms J, Kawahara N, Mazel C, et al. Review of metastatic spine tumour classification and indications for surgery: the consensus statement of the Global Spine Tumour Study Group. Eur Spine J. 2010;19:215-22.

30. Kato S, Hozumi T, Takeshita K, Kondo T, Goto T, Yamakawa K. Neurological recovery after posterior decompression surgery for anterior dural compression in paralytic spinal metastasis. Arch Orthop Trauma Surg. 2012;132:765-71. 